\title{
The total hospital and community UK costs of managing patients with relapsed breast cancer
}

\author{
RJ Thomas ${ }^{*, 1,2,3}$, M Williams', C Marshall', J Glen ${ }^{3}$ and M Callam' \\ 'The Primrose Oncology Research Unit, Bedford Hospital NHS Trust, Bedford, MK42 9Dj, UK; ' Department of Oncology, Addenbrooke's Hospital, \\ Cambridge University NHS Trust, Cambridge CB2 2QQ, UK; ${ }^{3}$ Cranfield Health, Cranfield University, Silsoe, Bedfordshire MK43 OAL, UK
}

The complete hospital and community records of 77 women were randomly selected from 232 women who had relapsed breast cancer between 2000 and 2005. Scrutiny of all management activities revealed a total cost of $£$ I 939329 (mean per patient of $£ 25$ । 86, 95\% Cl £|3705-£3382I). The median survival from time of relapse was 40.07 months and the median total cost per patient was $£ 31402.62$. Including the community cost of a relapse provides a more realistic figure for future cost-effectiveness analysis of adjuvant breast cancer therapies.

British Journal of Cancer (2009) I 00,598-600. doi: I0.1038/sj.bjc.66049II www.bjcancer.com

(c) 2009 Cancer Research UK

Keywords: cost; breast cancer; relapse

Developments in adjuvant drugs such as herceptin, aromatase inhibitors and taxanes, although initially expensive, reduce the risk of relapse and hence have long-term financial benefits (Thomas et al, 2006). To decide whether the benefits are cost-effective, budget holders require an accurate knowledge of how much it costs to treat patients with relapse. Most earlier estimates have used predictive modelling or interviews with clinicians (Mansel et al, 2007). A UK trial, now 6 years old, estimated hospital costs of relapsed breast cancer by sending questionnaires to UK oncologists and produced a figure of $£ 12500$ per patient (Remak and Brazil, 2004). A study from Edinburgh, collecting hospital data from node-positive women, gave a figure of $£ 13533$ for locoregional relapse and $£ 13193$ for distant relapse (Karnon et al, 2007). We report the first analysis of the total hospital and community cost of managing patients with relapsed breast cancer from a typical UK breast cancer practice.

\section{METHODS}

The Bedford Breast Cancer database identified 232 women who had relapsed between March 2000 and March 2005 with locoregional or distant disease. The identification codes were placed in separate sealed envelopes and one-third (77) randomly selected for data collection until their death or the predetermined end of the study in January 2007. The average age of the cohort was 62.3 years $(33-95)$ and $44 \%$ were originally node positive; $67 \%$ postmenopausal, $48 \%$ stage 1 (T1), 33\% T2, 16\% T3-4; 55\% oestrogen receptor $(\mathrm{ER})^{+\mathrm{ve}}, 25 \% \mathrm{ER}^{-\mathrm{ve}}(25 \%$ unknown $)$ and $21 \%$ overexpressed human epithelial growth factor receptor (HER)2 (17\% unknown). The demographics of the entire group and selected cohort were similar, indicating that the randomisation process was balanced. The specific cancer-related activities were derived from

*Correspondence: Dr RJ Thomas; E-mail: rt@cancernet.co.uk

Received 2 July 2008; revised 7 January 2009; accepted I I January 2009 written and electronic hospital records (Table 1). For each patient, a member of the research team visited all GP practices, hospices and community offices to collect all the relapse-related management and drug activities, which ensured there was no missing data. Once complete, the data set was locked and analysed in liaison with the Health Economics Department of Cranfield University. The predetermined subgroups chosen were menopausal status, ER status and HER2 expression, as these influence drug strategies on relapse. Whether patients suffered local or distant relapse was not included, as an earlier analysis did not demonstrate a cost difference (Karnon et al, 2007).

The NHS tariffs for outpatient visits, procedures, nights in hospital and investigations were derived from the existing NHS Trust Reference Cost Index 2004 (DoH, 2004). Radiotherapy was costed per fraction taken from the Reference Costs' National Average Unit Cost. General practitioner and district nurse visit from the PCT Reference Cost Index (DoH, 2004). Hospital drug costs included VAT and were the actual amount charged for each. Community drugs (excluding VAT) and hospice (including VAT) drug tariffs were taken from the British National formulary (BNF, 2005).

\section{RESULTS}

Of the 77 patients analysed in this study, 52 (67.5\%) had died of metastatic breast cancer by the end of the data-collection point, January 2007, with a median survival of 40.1 months (Figure 1). The first site of relapse was distant in 51 patients (66\%) and locoregional in $26(34 \%)$. The average time from initial diagnosis to relapse was 71.2 months (range $4-173$ months).

The total hospital and community cost of managing the 77 patients from relapse until death, or to the end of the evaluation period, was $£ 1939329$ (mean $£ 25$ 186, 95\% CI £13 705-£33 821). Dividing the total cost by the total number of patient months within the assessment period (2474.6) gave an average monthly 
Table I Categories of costs following relapse (77 patients)

\begin{tabular}{|c|c|c|c|c|c|}
\hline & Source & $95 \%$ & Confidence interval & Mean & Total \\
\hline GP home visit & GPDB & 301 & 657 & 480 & 36950 \\
\hline Hospice nights & GPBD & 495 & 1796 & 1146 & 88241 \\
\hline Hospice visits & $\mathrm{HDB}$ & 25 & 118 & 72 & 5554 \\
\hline Palliative community telephone & $\mathrm{HBD}$ & 39 & 86 & 63 & 4824 \\
\hline GP-prescribed drugs & GPDB & 2350 & 4481 & 2916 & 263036 \\
\hline Hospice drugs & $\mathrm{HN}$ & 70 & 614 & 272 & 20954 \\
\hline Sub-total community costs & & 4479 & 10903 & 7126 & $£ 587195$ \\
\hline Percentage total cost (£| 939 329) & & & & & $30.3 \%$ \\
\hline \multicolumn{6}{|l|}{ Hospital drugs } \\
\hline Outpatients' hospital pharmacy & PDB & 3839 & 7940 & 5890 & 453495 \\
\hline \multicolumn{6}{|l|}{ Hospital bed costs } \\
\hline Surgical interventions & PIMS & 733 & 1489 & 1112 & 85603 \\
\hline Blood transfusion & $\mathrm{HN}$ & 372 & 1044 & 708 & 54534 \\
\hline \multicolumn{6}{|l|}{ Outpatients' visits } \\
\hline Outpatient consultation & PIMS & $247 \mid$ & 3602 & 3037 & 233849 \\
\hline Day/half-day case & PIMS & 1040 & 1981 & $151 \mid$ & 116352 \\
\hline ECG & DR HN & 41 & 83 & 63 & 4842 \\
\hline Radiotherapy & RTDB & 408 & 764 & 587 & 45166 \\
\hline Support services & $\mathrm{DR} H \mathrm{HN}$ & 48 & 106 & 78 & 5981 \\
\hline $\mathrm{A}$ and $\mathrm{E}$ admissions & PIMS & 129 & 213 & 171 & 13208 \\
\hline \multicolumn{6}{|l|}{ Hospital services } \\
\hline Radiology & DDB & 554 & 856 & 705 & 54322 \\
\hline Pathology & DDB & 292 & 459 & 376 & 28967 \\
\hline
\end{tabular}

$\mathrm{DDB}=$ departmental database; $\mathrm{DR}=$ departmental written records; $\mathrm{GPDB}=$ general practice database; $\mathrm{HN}=$ hospital notes; $\mathrm{INR}=$ individual nursing records; $\mathrm{PDB}=$ pharmacy database; PIMS = Patient Information Management System; RTBD = Addenbrooke's oncology electronic database

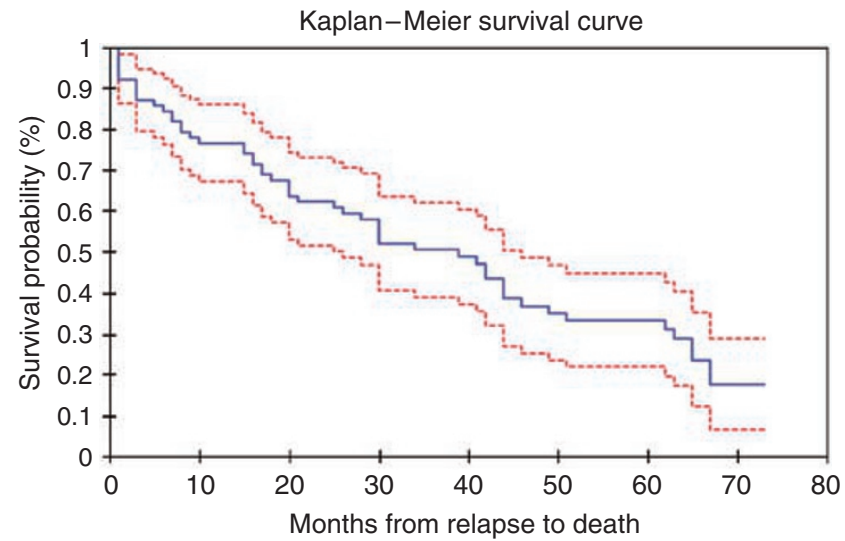

Figure I Kaplan-Meier life table and survival curve for 77 patients from date of relapse. Median survival 40.07 months (25 were alive at the time of analysis).

cost of $£ 783.7$ per month. The median cost per patient derived from the basic data was $£ 19886.6$. The median cost per patient taking into account that not all patients died during the evaluation period was also estimated by multiplying the monthly average by the median survival (40.07), which gave a figure of $£ 31402.62$.

Nearly a third $(30.3 \%)$ of the cost of a relapse lay in the community and $69.7 \%$ in the hospital setting. The total drug cost was $39 \%$ of the total. There was a non-statistically significant trend for patients who were pre-menopausal (32), being subsequently more expensive to manage on relapse than post-menopausal (45) (ratio of mean cost per patient 1.44:1). Likewise, $\mathrm{ER}^{-\mathrm{ve}}$ women were more expensive to treat on relapse than $\mathrm{ER}^{+ \text {ve }}$ (61) (ratio $1.34: 1)$. Women with tumours overexpressing HER2 were more expensive to treat than HER2 normal (48) or those not tested (13) (ratio $1.25: 1$ ). In this study cohort, only 7 of the 16 received herceptin on relapse. There was no trend between those axillary nodes positive (35) compared with those negative (21) or unknown (21) (ratio $1.04: 1$ ).

\section{DISCUSSION}

The strength of this study lies in the comprehensiveness of the data extracted from a typical breast cancer population treated within a standard UK management framework. Collecting the data from both hospital and community sources required substantial co-operation from a wide range of professional groups, and a 
single institution ameliorated the absence of missing data. This advantage, however, is also the basis of a potential criticism if this data were to be extrapolated nationally, as a single-institution study may have been subject to skews in local demographics or medical practice.

The demographics of the North Bedfordshire population, obtained from 2003 government figures, however, appeared fairly typical for the United Kingdom (Census, 2001). The average age was in line with the national average as was the single, separated and widowed population. The percentages of people with British citizenship (91.4 vs $87.5 \%$ ) and those born in the United Kingdom (93.0 vs 91.1\%) were similar. There was a slightly higher percentage of the population working (42.6 vs $40.1 \%$ ), and fewer unemployed (2.6 vs 3.4\%), but a similar percentage who achieved higher educational qualifications (18.9 vs 19.8\%). Breast cancer management adhered to West Anglia Cancer Network guidance, which had been developed from national advisory documents. Any deviation from the guidance required formal documentation through a concession form system.

The median overall survival for the patients relapsing at our institution (40.7 months) was higher than the 10-20 months reported in first-line metastatic studies in the United Kingdom and Europe evaluating metastatic chemotherapy regimens (Bontenbal et al, 2005), probably explained by our group including $30 \%$ with loco-regional relapse only. Overall survival was, however, comparable with the 20-30 months reported within first-line metastatic aromatase vs tamoxifen studies (Nabholtz et al, 2000).

The median figure of $£ 31402.6$ for treating a relapse is higher than previous UK estimates and this is largely due to the $30.3 \%$ of community costs not collected earlier. Even though this data set is

\section{REFERENCES}

Bontenbal M, Creemers GJ, Braun HJ, Senaeve C (2005) Phase II to III study comparing doxorubicin and docetaxel with fluorouracil, doxorubicin, and cyclophosphamide as first-line chemotherapy in patients with metastatic breast cancer. J Clin Oncol 23(28): 7081-7088

BNF (September 2005), No. 50, ISBN 978-0-85369-778-7

Census (2001) County Profile - Bedfordshire Crown Copyright 2003

DoH (2004) NHS Trust Reference Cost Index 2004 published on the DoH website http://www.dh.gov.uk/en/Publicationsandstatistics/Publications/ PublicationsPolicyAndGuidance/DH 4105545

Karnon J, Kerr GR, Jack W, Papo NL, Cameron DA (2007) Health care costs for the treatment of breast cancer recurrent events: estimates from a UK-based patient-level analysis. Br J Cancer 97: 479-485

Mansel R, Locker G, Fallowfield L, Benedict A, Jones D, on behalf of the ATAC Trialists' Group (2007) Cost-effectiveness analysis of anastrozole $v s$ tamoxifen in adjuvant therapy for early stage breast cancer in the United Kingdom: the 5-year completed treatment analysis of the ATAC the most up to date at the time of publication, these figures represent the last 6 years, and are therefore still likely to underestimate future costs. The full impact of herceptin, for example, was not fully appreciated. Routine use of herceptin in the metastatic setting within our institution was introduced in November 2006, almost halfway through the evaluation period. This meant that only 7 of the 16 HER2-overexpressing patients received herceptin as part of their metastatic management. The average cost of herceptin in treated patients was £15834.6. According to the original published herceptin metastatic trial data, these patients, on average, could have lived 5 months longer (Vogel et al, 2002). In theory, therefore, if the remaining nine patients had received herceptin and each lived 5 months longer, this factor alone would have increased the total cost to over $£ 34200$ per patient.

There is no doubt that newer biological agents, such as oral tyrosine kinase inhibitors and antiangiogenesis drugs, will also substantially add to the metastatic drug costs and as they will keep relapsed patients alive for longer, this will also increase their nondrug costs. The authors intend to repeat the same data-collection exercise every 2 years using the same methodology to give an ongoing estimate of the cost of a relapse, as these newer biological agents evolve into clinical practice. In the mean time, these data by including the community cost improve the accuracy of the costeffectiveness analysis of adjuvant breast cancer therapies.

\section{ACKNOWLEDGEMENTS}

This study was supported by Pfizer, Sanofi Aventis, Astra Zeneca and Roche.
('Arimidex', Tamoxifen alone or in combination) trial. Br J Cancer 97: $152-161$

Nabholtz JM, Buzdar A, Pollak M, Harwin W, Burton G, Mangalik A, Steinberg M, Webster A, von Euler M (2000) Anastrozole is superior to tamoxifen as first-line therapy for advanced breast cancer in postmenopausal women: results of a North American multicenter randomized trial. J Clin Oncol 18: $3758-3767$

Remak E, Brazil L (2004) Cost of managing women presenting with stage IV breast cancer in the United Kingdom. Br J Cancer 91: 77-83

Thomas R, O'Sullivan B, Glen J, Callam M (2006) Adjuvant breast cancer drugs - blockbusters or bankrupters? Br J Cancer Manage 3(24): 5-9

Vogel CL, Cobleigh MA, Tripathy D, Gutheil JC, Harris LN, Fehrenbacher L, Slamon DJ, Murphy M, Novotny WF, Burchmore M, Shak S, Stewart SJ, Press M (2002) Efficacy and safety of Trastuzumab as a single agent in first-line treatment of HER2-overexpressing metastatic breast cancer. J Clin Oncol 20(3): 719-726 